\title{
EQUIVALENCE OF THE CLASSICAL THEOREMS OF SCHOTTKY, LANDAU, PICARD AND HYPERBOLICITY
}

\author{
KYONG T. HAHN ${ }^{1}$
}

\begin{abstract}
Modifying the classical theorems of Schottky and Landau, the author obtains the converses of these theorems. More precisely, the author defines the notions of Schottky, Landau and Picard properties and proves that a plane domain $D$ satisfies any of these properties if and only if $\mathbf{C} \backslash D$ contains at least two points. The method of proofs is completely elementary and uses only some basic properties of the Kobayashi metric.
\end{abstract}

1. Introduction. The main object of this note is to formulate the classical theorems of Schottky and Landau in their strongest forms so that the converses of these theorems are also valid.

A domain $D$ in the complex plane $\mathbf{C}$ is said to satisfy the Schottky property if for each $w_{0} \in D$, a bounded neighborhood $W$ of $w_{0}$ in $D$ with $\bar{W} \subset D$ and an $r \in(0,1)$, there exists a positive constant $\Omega=\Omega(W, r)$ such that every holomorphic mapping $f$ of the open unit disc $\Delta$ into $D$ with $f(0) \in W$ satisfies $|f(z)| \leqslant \Omega$ for $|z| \leqslant r$.

Similarly, a domain $D \subset \mathrm{C}$ is said to satisfy the Landau property if for each $w_{0} \in D$ and a bounded neighborhood $W$ of $w_{0}$ with $\bar{W} \subset D$ and any $a>0$, there exists a positive constant $R=R(W, a)$ such that if $\left|f^{\prime}(0)\right| \geqslant a r$ for every holomorphic mapping $f: \Delta \rightarrow D$ with $f(0) \in W$, then $r \leqslant R$.

Finally, we also define the Picard property as well and make some trivial observation about the little Picard theorem.

A domain $D \subset \mathbf{C}$ is said to have the Picard property if every holomorphic mapping $f: \mathbf{C} \rightarrow D$ reduces to a constant mapping. It is the content of the little Picard theorem that a domain with at least two boundary points has the Picard property. The converse of this theorem also holds for a plane domain. Namely, every plane domain having the Picard property must have at least two boundary points.

The result of this note is then given by the following

THEOREM. For a domain $D \subset \mathbf{C}$, the following statements are equivalent.

(a) D has at least two boundary points.

(b) D satisfies the Schottky property.

(c) D has the Landau property.

(d) D has the Picard property.

Received by the editors December 8, 1982. This paper was presented at the eighty-sixth summer meeting of the AMS in August 23, 1982.

1980 Mathematics Subject Classification. Primary 30F 10, 32H20; Secondary: 30C 99.

Key words and phrases. Classical theorems of Schottky and Landau, Schottky-property, Landau-property, Picard-property, Kobayashi metric, hyperbolicity, Poincaré metric.

${ }^{1}$ Research partially supported by NSF Grant MCS 80-02915 at Pennsylvania State University. $0002-9939 / 83 \$ 1.00+\$ .25$ per page 
We remark that the Picard property is in general a weaker notion than the rest of the conditions in the Theorem for higher dimensional case [2]. Using the notions of Kobayashi metric and hyperbolicity, we give here an elementary proof of this theorem.

2. Kobayashi pseudometric and hyperbolicity. In [3], S. Kobayashi has introduced the notion of the so-called Kobayashi pseudometric on a complex manifold. Here we give a brief review of this notion on a plane domain $D$.

Given a pair of points $p$ and $q$ in $D$, we choose a chain $\alpha$ connecting $p$ and $q$ in $D$ by taking points $p=p_{0}, p_{1}, \ldots, p_{l-1}, p_{l}=q$ in $D$, points $a_{1}, \ldots, a_{l}$ in $\Delta$ and holomorphic mappings $f_{1}, \ldots, f_{l}$ of $\Delta$ into $D$ such that $f_{i}(0)=p_{i-1}, f_{i}\left(a_{i}\right)=p_{i}(i=1, \ldots, l)$. Then the Kobayashi pseudometric on $D$ is defined by

$$
k_{D}(p, q)=\inf _{\alpha}|\alpha|, \quad|\alpha|=\sum_{i=1}^{l} \rho_{\Delta}\left(0, a_{i}\right),
$$

where the infimum is taken over all chains $\alpha$ connecting $p$ and $q$, and $\rho_{\Delta}$ denotes the Poincaré metric on $\Delta$, i.e.,

$$
\rho_{\Delta}(0, z)=\frac{1}{2} \log \frac{1+|z|}{1-|z|}=\tan h^{-1}|z|, \quad z \in \Delta .
$$

It is a simple matter to check that $k_{D}$ is a pseudometric. In fact, the Kobayashi pseudometric for the complex plane $\mathbf{C}$ is trivial, i.e., $k_{\mathbf{C}}(p, q) \equiv 0$ for $p, q \in \mathbf{C}$. To see this, let $p$ and $q$ be any two points in $\mathbf{C}$ and let $n>|q-p|$ be an integer. Connect $p$ and $q$ by the chain $\alpha_{n}$ with $l=1$ by taking $a_{1}=(q-p) / n \in \Delta$ and $f_{1}(z)=n z+p(z \in \mathbf{C})$ so that $f_{1}(0)=p, f_{1}\left(a_{1}\right)=q$ and

$$
k_{\mathbf{C}}(p, q) \leqslant \rho_{\Delta}(0,(q-p) / n) \rightarrow 0 \text { as } n \rightarrow \infty .
$$

Among many properties that the Kobayashi pseudometric enjoys, the following two properties are more basic (see [3]).

Proposition 1. If $f: D \rightarrow G$ is a holomorphic mapping of a domain $D$ into another domain $G \subset \mathbf{C}$, then

$$
k_{G}(f(p), f(q)) \leqslant k_{D}(p, q) \text { for } p, q \in D .
$$

In particular, if $D \subset G$ then

$$
k_{G}(p, q) \leqslant k_{D}(p, q) \quad \text { for } p, q \in D .
$$

If $f$ is a biholomorphic mapping of $D$ onto $G$, then

$$
k_{G}(f(p), f(q))=k_{D}(p, q) \text { for } p, q \in D .
$$

Proposition 2. The Kobayashi metric coincides with the Poincare metric at every point of the unit disc $\Delta$.

Following S. Kobayashi [3], we define a domain $D \subset \mathbf{C}$ to be "hyperbolic" if the Kobayashi pseudometric $k_{D}$ is a metric. Therefore, any disc is hyperbolic in the sense of Kobayashi while the complex plane and the Riemann sphere (= extended complex plane) are not. 
In [4], H. Royden has constructed the infinitesimal form of the Kobayashi metric:

$$
K_{D}(p, \xi)=\inf \left\{\frac{|\xi|}{\left|f^{\prime}(0)\right|}: \exists f \in H(\Delta, D) \ni f(0)=p\right\}, \quad p \in D,
$$

$\xi \in \mathbf{C}$, where $H(\Delta, D)$ denotes the set of all holomorphic mappings $f: \Delta \rightarrow D$, and has shown that the Kobayashi metric $k_{D}$ is the integrated form of $K_{D}$. The notion of hyperbolicity of $D$ can also be defined in terms of $K_{D}$ : A domain $D$ is hyperbolic if for each $p \in D$ there exists a neighborhood $U$ of $p$ and a positive constant $C$ such that

$$
K_{D}(q, \eta) \geqslant C\|\eta\| \quad \text { for } q \in U \text { and } \eta \in \mathbf{C} .
$$

Then this definition coincides with the hyperbolicity in the sense of Kobayashi (see [4]). Therefore, it follows that this definition of hyperbolicity actually coincides with the hyperbolicity in the classical sense for a Riemann surface. By the uniformization theorem, every Riemann surface has an essentially unique universal covering surface which is either conformally equivalent to a disc, to the complex plane or to the Riemann sphere. A Riemann surface of hyperbolic type in the classical sense is then the one whose universal covering surface is equivalent to a disc. On the other hand, the Kobayashi hyperbolicity is preserved under covering maps. More precisely, we have

Proposition 3. A Riemann surface $M$ is hyperbolic in the sense of Kobayashi if and only if its universal covering surface $M$ is.

This proposition has been proved in [3] for complex manifolds.

The following Corollary is an immediate consequence of Proposition 3 when we observe that any plane domain having one boundary point has $\mathbf{C}$ as its universal covering surface.

COROLlARY. On a plane domain $D$ the following statements are equivalent.

(a) D has at least two boundary points.

(b) $D$ is hyperbolic in the classical sense.

(c) $D$ is hyperbolic in the sense of Kobayashi.

3. Proof of the Theorem. (a) $\Rightarrow(b)$ : Assume that $D$ has at least two boundary points. By the Corollary, $D$ is hyperbolic and has complete Poincare metric $\rho_{D}$ which agrees with Kobayashi metric $k_{D}$. Let $w_{0} \in D$ and $W$ be any bounded neighborhood of $w_{0}$ with $\bar{W} \subset D$. Since $\rho_{D}$ is a complete metric, we may set $W=\{w \in D$ : $\left.\rho_{D}\left(w_{0}, w\right)<\rho_{0}\right\}$ for some $\rho_{0}>0$. Let $U=\left\{w \in D: \rho_{D}\left(w_{0}, w\right)<\rho_{0}+\rho_{1}\right\}$, where $\rho_{1}=\tan h^{-1} r$ with $r \in(0,1)$.

By the triangle inequality,

$$
\rho_{D}\left(w_{0}, f(z)\right) \leqslant \rho_{D}\left(w_{0}, f(0)\right)+\rho_{D}(f(0), f(z)) .
$$

If $f \in H(\Delta, D)$ satisfies $f(0) \in W$, then

$$
\rho_{D}\left(w_{0}, f(0)\right)<\rho_{0} .
$$


If, in addition $|z| \leqslant r$, then

$$
\rho_{D}(f(0), f(z)) \leqslant \rho_{\Delta}(0, z) \leqslant \tan h^{-1} r=\rho_{1} .
$$

From (1), (2) and (3),

$$
\rho_{D}\left(w_{0}, f(z)\right) \leqslant \rho_{0}+\rho_{1} .
$$

Namely, $f(z) \in U$ whenever $|z| \leqslant r$. Since $\bar{U}$ is compact in $D$ with respect to $\rho_{D}$, it is contained in a bounded set of $\mathbf{C}$. Therefore, there exists a positive number $\Omega$ which depends only on $W$ and $r \in(0,1)$ such that $|f(z)| \leqslant \Omega$ for $|z| \leqslant r$.

(b) $\Rightarrow(a)$ : We assume that $D$ has the Schottky property. By the corollary to Proposition 3, it is enough to prove that $D$ is Kobayashi hyperbolic. Given $w \in W$ and $\eta \in \mathbf{C}$, let $f: \Delta \rightarrow D$ be a holomorphic map with $f(0)=w$. By the Cauchy estimate for $f$ on $|z| \leqslant r,\left|f^{\prime}(0)\right| \leqslant \Omega / r$ or $|\eta| /\left|f^{\prime}(0)\right| \geqslant(r / \Omega)|\eta|$. Therefore,

$$
K_{D}(w, \eta) \geqslant(r / \Omega)|\eta|,
$$

for all $w \in W$ and $\eta \in \mathbf{C}$, which implies that $D$ is hyperbolic in the sense of Kobayashi.

(a) $\Rightarrow$ (c): If $D$ has at least two boundary points, then $D$ can be furnished with the Poincare metric $\rho_{D}$. Suppose that $D$ fails to satisfy the Landau property. Then there exists a point $w_{0} \in D$, its open neighborhood $W$ with $\bar{W} \subset D$, some $a>0$, a sequence $\left\{r_{k}\right\}$ of positive real numbers tending to $\infty$ and a sequence $f_{k} \in H(\Delta, D)$ such that $f_{k}(0) \in W$ and $\left|f_{k}^{\prime}(0)\right| \geqslant a r_{k}$ for all $k$. We claim that if $D$ has the Poincaré metric, then there is an $r \in(0,1)$ such that the sequence $\left\{f_{k}\right\}$ contains a subsequence which converges uniformly to a holomorphic mapping $f: \Delta_{r} \rightarrow D$. Since $\bar{W}$ is compact in $D$, there exists a number $\rho^{\prime}>0$ such that

$$
Q=\left\{w \in D: \rho_{D}(\bar{W}, w)<\rho^{\prime}\right\} \subset D .
$$

For all $f_{k} \in H(\Delta, D)$ with $f_{k}(0) \in W$,

$$
\rho_{D}\left(f_{k}(0), f_{k}(z)\right) \leqslant \rho_{\Delta}(0, z)<\rho^{\prime},
$$

so that $f_{k}(z) \in Q$ whenever $|z| \leqslant r^{\prime}=\tan h \rho^{\prime}$. Since $\bar{Q}$ is compact, it is bounded. Therefore, by Montel's theorem, there exists a subsequence of $\left\{f_{k}\right\}$ which converges uniformly on $\Delta_{r}$ to a holomorphic mapping $f: \Delta_{r} \rightarrow D$ for $r<r^{\prime}$. Denote this convergent subsequence again by $\left\{f_{k}\right\}$. Now, by the theorem of Weierstrass, $f_{k}^{\prime}(z)$ converges to $f^{\prime}(z)$ uniformly on $\Delta_{r}$. In particular, $f_{k}^{\prime}(0)$ converges to $f^{\prime}(0)$ which contradicts the fact that $\left|f_{k}^{\prime}(0)\right| \rightarrow \infty$ as $k \rightarrow \infty$.

(c) $\Rightarrow$ (a): Suppose that $D$ has the Landau property. Then for each $p \in D$ and each bounded neighborhood $W$ of $p$ in $D$, there exists a positive number $R_{1}=R_{1}(W)$ such that

$$
\sup \left\{\left|f^{\prime}(0)\right|: f \in H(\Delta, D) \text { and } f(0) \in W\right\} \leqslant R_{1}<\infty .
$$

Let $\xi \in \mathbf{C}$. For each $q \in W$, let $f \in H(\Delta, D)$ satisfy $f(0)=q$. Then by (6)

$$
\frac{|\xi|}{\left|f^{\prime}(0)\right|} \geqslant \frac{1}{R_{1}}|\xi|
$$


which implies

$$
K_{D}(q, \xi) \geqslant C|\xi| \quad\left(C=1 / R_{1}>0\right)
$$

for $q \in W$ and $\xi \in \mathbf{C}$, and the hyperbolicity of $D$.

(a) $\Leftrightarrow$ (d): It follows trivially from the little Picard theorem and the remark given in $\S 1$.

\section{REFERENCES}

1. D. Bridges, A. Calder and W. Julian, Picard's theorem, Trans. Amer. Math. Soc. 209 (1982), 513-530.

2. K. T. Hahn and K. T. Kim, Hyperbolicity of a complex manifold, and other equivalent properties, preprint.

3. S. Kobayashi, Hyperbolic manifolds and holomorphic mappings, Pure and Appl. Math. 2, Dekker. New York, 1970.

4. H. Royden, Remarks on the Kobayashi metric, Several Complex Variables, II (Proc. Internat. Conf., Univ. Maryland, College Park, Md., 1970). Lecture Notes in Math., vol. 185. Springer-Verlag, Berlin. 1971, pp. 125-137.

5. W. A. Veech, A second course in complex analysis, Benjamin, New York, 1967.

Department of Mathematics, Pennsylvania State University, University Park, Pennsylvania 16802 